\title{
Interacciones comunicativas a través de Internet.
}

\author{
Blanca Chong López*
}

\section{Introducción.}

El propósito del presente trabajo es abordar algunas reflexiones acerca de cómo Internet se ha convertido en un medio que está transformando la cultura actual, por la influencia que tiene en la vida cotidiana de millones de seres humanos, pero sobre todo, por la posibilidad que brinda de nuevas formas de interacción comunicativa.

El desarrollo de las Tecnologías de Información, especialmente Internet, representa un enorme desafío para los estudiosos de lo social, pues supone transformaciones que nos llevan a una nueva cultura, a una "sociedad red" (Castells, 2001a) en donde se modifican formas de trabajo, de entretenimiento, de educación y cultura, pero sobre todo y de manera muy importante, las formas de interacción social, y por lo tanto comunicativa. Para Castells "internet es -y será aún más- el medio de comunicación y de relación esencial sobre el que se basa una nueva forma de relación que ya vivimos." (p.50).

Internet modifica las maneras en que la gente se relaciona entre sí, al hacer posibles nuevas formas de interacción entre los individuos, en donde el tiempo y el espacio adquieren dimensiones distintas a las que han tenido hasta hoy. Actualmente podemos hablar del homo internetus, del hombre que vive on line.

Al referirse a la trascendencia de este medio para la sociedad, González señala:

La posibilidad de viajar por el "ciberespacio", acceder a miles de bases de datos de la más variada naturaleza y entrar en contacto con millones de usuarios de remotos países, se ha convertido en una enajenante adicción para muchas personas y también en el ideal nunca antes soñado de técnicos y especialistas, publicitarios y comerciantes, en todo el mundo. (González, 1998, p.99).

En cuanto a los usuarios de Internet, los dos sectores que los especialistas de la sociedad de la información consideran más próximos a su aceptación, son por una parte los jóvenes y los niños (aspecto generacional), y por otra los empresarios (aspecto

* Universidad Autónoma de Coahuila 
económico). Los niños y jóvenes son los más proclives a comprender las ventajas que supone su uso. Para las empresas la motivación reside en la eficacia. (mayor productividad, menores costos, menos personal, mejor información). (Joyanes, 1997, p.35)

\section{¿Por qué estudiar lo relacionado con Internet?.}

Aunque en México desde el surgimiento de Internet varios estudiosos de la comunicación se han ocupado del tema, es aún poco lo que conocemos sobre la cibercultura en nuestro medio. Actualmente la mayor parte de los estudios empíricos acerca de Internet siguen realizándose en Estados Unidos.

Coincido con Castells, quien cuando habla de la necesidad de considerar Internet como objeto de estudio, señala que:

es el tejido de nuestras vidas. Si la tecnología de información es el equivalente histórico de lo que supuso la electricidad en la era industrial, en nuestra era podríamos comparar a Internet con la red eléctrica y el motor eléctrico, dada su capacidad para distribuir el poder de la información por todos los ámbitos de la actividad humana. (Castells, 2001c: 15).

La actividad humana se basa en la comunicación e Internet transform a elm odo en que nos cam unicam os.'

La refuncionalización de la comunicación a partir de la integración de Internet en la vida social es un factor de gran relevancia para los estudios sobre la comunicación y la cultura. "No se trata sólo de la aparición de un nuevo medio sino de un cambio mayor". (Fuentes, 2001: 233). Es necesario reformular los objetos de estudio

'Para tener una idea de la manera en que esta herramienta se ha incorporado a la vida cotidiana, debemos considerar que en 1972 solamente había ciento cincuenta mil computadoras en el mundo. (Negroponte, 1996). Para la mayoría de la sociedad Internet nació en 1995, a finales de ese año, el primero de uso generalizado de la red, había unos 16 millones de usuarios en todo el mundo. A principios de 2001 ya había más de $\mathbf{4 0 0}$ millones, y las predicciones consideran que el número de usuarios para 2005 llegará a 1,000 millones. Es posible que para el año 2010 se alcance la cifra de 2,000 millones, incluso teniendo en cuenta el desarrollo desigual de esta tecnología en el mundo. (Castells, 2001c: 17).

Otras cifras nos hablan de que hacia mediados de 2001 existían casi 30 millones de sitios web. Si se considera que en 1993 había solamente 130 sitios, que en 1996 sumaban 25 mil y en 2000 el número había crecido a 17 millones, podemos tener una idea del importante crecimiento que ha tenido Internet. (Trejo, 2002a) 
considerando los cambios en las relaciones socioculturales, en la organización de la vida cotidiana y de sus representaciones cognitivas, en la distribución de las posiciones de poder y en las dimensiones de espacio y tiempo en que se sitúa la actividad humana.

Los nuevos espacios de comunicación significan para algunos estudiosos la llegada a la cúspide de la interacción y la estructura social, afirmaciones que resultan arriesgadas. Considero que si bien el desarrollo tecnológico en el campo de la información tiene una gran trascendencia, por lo que se ha señalado, es necesario conocer con mayor profundidad las formas de interacción que establecen los individuos en la comunicación mediada por computadora, para poder sostener que se trata de la mayor interacción posible. En cuanto a la transformación de la estructura social, para que ésta se dé son necesarios cambios que van más allá de lo tecnológico.

Hay quienes consideran que a partir de los cambios científicos, tecnológicos y sociales ocurridos a mediados de la década de los noventa, se está transitando de una sociedad de la información hacia una sociedad de la comunicación y el conocimiento. (Pineda, 2003 y Galindo, 1998). En esta nueva sociedad, señala Pineda, la información es sólo un elemento que activa el proceso, la comunicación es el nexo que favorece la relación entre individuos y el conocimiento es lo que permite generar valor agregado a la información y desarrollar las potencialidades de la inteligencia humana. Lo importante serían las relaciones entre los sujetos, no la relación técnica sujeto-objeto.

La sociedad de la información se organiza con base en relaciones y estructuras rígidas, que inhiben la creación y la iniciativa; la sociedad de la comunicación cuenta con estructuras tanto horizontales como verticales, lo que permite el acuerdo e interacción entre pares. La sociedad del conocimiento sería el espacio donde el hombre busca desarrollar la comunicación, la interacción entre personas y no sólo proporcionar información; busca fomentar el aprendizaje y desarrollo de la inteligencia. La tecnología en este tipo de sociedad deberá ser concebida como sistema socio-técnico, que refiere a nuevas formas de organización social. Los cambios que han ocurrido, por tanto, no son solamente técnicos, sino principalmente sociales y culturales. En este punto es necesario insistir en que tendríamos que revisar qué elementos definen una sociedad, para poder hablar de un nuevo tipo de sociedad, discusión que va más allá de los propósitos de este trabajo.

Sin Internet las relaciones de comunicación a distancia no habrían tenido el crecimiento que hemos visto en los últimos años, lo cual ha 
dado origen al concepto de "ciberespacio", que hace referencia no sólo a las conexiones tecnológicas, sino al espacio de relaciones y contactos humanos entre los usuarios, que a largo plazo podría modificar las formas de comunicación entre las personas.

\section{Las visiones pesimistas acerca de Internet.}

No todos los estudiosos de la nueva realidad comunicacional ven con el mismo optimismo la inserción de Internet en la vida social. Para Ramonet, quien mantiene una visión un tanto escéptica acerca de este medio, cuando se produce un cambio importante en el campo de la comunicación, éste se presenta como la posibilidad de acceder a un mundo mejor; ha ocurrido con el telégrafo, la radio, la televisión, el teléfono, y lo mismo pasa con Internet. Debemos preguntarnos qué sucede cuando se pretende hacer llegar Internet a donde no existen condiciones mínimas de desarrollo:

llega Internet como un marciano en un territorio vacío. Eso no quiere decir que Internet no tenga potencialidades. Pero la ilusión de que Internet por sí misma va a transformar el mundo y va a hacer que la gente pase de una era medieval al futuro se demostró que no funciona. (Ramonet, 2001: 56).

Algunos argumentos para mantener esa visión escéptica sobre Internet, son, por ejemplo, que a pesar de la sencillez de su uso se requiere por lo menos estar alfabetizado, mientras que para el uso de la radio o la televisión no era necesaria preparación alguna. La iniciación en Internet, por mínima que sea, significa en muchos casos un enorme esfuerzo. Existen países, como Marruecos, donde el $60 \%$ de la población es analfabeta. Según informe reciente de la UNESCO, solamente el $2.3 \%$ de la población mundial ha utilizado en algún momento Internet.

Es cierto que en el acceso a la tecnología existen enormes diferencias: En los países más desarrollados en América del Norte y Europa los usuarios de la red representaban en 2001 la cuarta parte de la población y en algunos llegaban a la mitad o más, en el resto del mundo el porcentaje era aún mínimo. (Trejo Delarbre, 2002b). Según el Programa de Naciones Unidas sobre el Desarrollo (PNUD), únicamente el $3 \%$ de la humanidad comparte el uso de las nuevas tecnologías, y de éste un 3\% corresponde a países subdesarrollados. (González, 2000). 
En el caso de México, el número de usuarios de Internet en el año 2000 era 36 veces mayor que el de cinco años atrás, sin embargo ese número representaba sólo $3.5 \%$ de la población total. Según el censo del 2000,8.6 millones de mexicanos disponían de computadora en su hogar, cifra que representaba menos del $10 \%$ de la población total. (Este País, 2001: 60)

Coincido con Castells cuando afirma que la comunicación a través de Internet seguirá siendo el dominio de sectores cultos de la población de los países más avanzados, que representan decenas de millones, pero sólo una elite a escala global. Este tipo de comunicación comienza como el medio utilizado por los segmentos más cultos de los países ricos. Sin embargo, en el futuro cercano se extenderá su uso, sobre todo a través del sistema educativo, y llegará a proporciones considerables de la población. Pero el hecho de que se expanda en olas sucesivas, a partir de una elite cultural, significa que será ésta quien determinará con sus usos los hábitos de la comunicación. (Castells, 2001b).

Crovi (2002), por su parte sostiene que la apropiación y uso de las nuevas tecnologías plantea numerosos interrogantes para el futuro de las relaciones sociales. Si bien reconoce que estas tecnologías están al alcance solamente de algunos grupos sociales, señala que por las ventajas que ofrecen su uso se va extendiendo a otros sectores sociales. Sería importante, tomando en cuenta esta opinión, analizar cómo individuos pertenecientes a los grupos menos favorecidos se han ido acercando al uso de las tecnologías de información. En la revisión que se ha hecho sobre el tema no se ha encontrado un estudio de esta naturaleza.

Otra mirada, que considero acertada en cuanto a lo que Internet representa para el futuro de la humanidad es la de Wolton, quien reconoce que actualmente la comunicación es fundamental en la sociedad, pero frente a la postura de los que identifican la sociedad del mañana con las tecnologías dominantes, señala que:

Historicamente hablando, no existe un tiempo que vaya de la tecnología a la sociedad, sino que es más bien lo contrario. La historia social, economica y cultural da normalmente sentido a la historia técnica y no al revés, y esto sucede a través de diacronfas diferentes. (1999: 14). 
Formula también las siguientes preguntas:

¿Por qué esta ingenua idea, según la cual la omnipresencia del ordenador y de la televisión cambiará radicalmente las relaciones humanas y sociales, se ha impuesto de un modo tan fuerte y atractivo? ¿Por qué asistimos a esta tecnificación de la comunicación y de la sociedad?. (1999: 15).

Comprender el papel de la comunicación en una sociedad significa analizar las relaciones fundamentales entre los tres aspectos principales de la comunicación: sistema técnico, modelo cultural dominante y proyecto que sobreentiende la organización económica, técnica y jurídica del conjunto de las técnicas de comunicación.

Actualmente un importante número de estudiosos de la comunicación considera que Internet es una verdadera revolución que dará lugar a una "nueva sociedad", es decir adoptan una postura de determinismo tecnológico. Con esto se refuerza la ideología tecnológica actual, que contempla la revolución en las tecnologías como condición para una revolución en las relaciones humanas y sociales. Para Wolton, el objetivo de estudiar Internet no es saber si todo el mundo se beneficiará de él, ni lo que permite hacer, sino comprender si existe alguna relación entre este sistema técnico y un cambio de modelo cultural y social de la comunicación.

La seducción que ejercen las nuevas tecnologías se explica a partir de una insuficiente valorización del concepto de comunicación y la desconfianza hacia los medios de comunicación, tan desproporcionada como la confianza en las nuevas herramientas.

Las nuevas tecnologías de comunicación resultan tan atractivas por la importancia que sobre todo para los jóvenes tiene la idea de apertura, pero además el rechazo a la omnipresencia de los medios masivos de comunicación, así como la atracción por la modernidad, entre otras razones. Las dimensiones psicológicas son también esenciales en esa atracción, porque las nuevas tecnologías favorecen el importante movimiento de individualización de nuestra sociedad.

Wolton critica además el que para muchos, el número de computadoras conectadas a Internet sea considerado como el indicio más preciso del grado de desarrollo de un país.

Por su parte, Román Gubern (2002) señala que si bien el uso del ordenador representa una gran revolución que no debemos subestimar, es preciso señalar que se trata de "una revolución en el primer mundo". 
Considera que es retórica decir que hoy se vive en una sociedad global, cuando solamente dos por ciento de la humanidad está conectada a Internet. $^{2}$

Para Gubern un efecto negativo de la computadora es que vuelve más sedentaria a la sociedad. El tele trabajo, el tele ocio, la tele escuela generan una sociedad que él denomina "claustrofílica", centrada en el hogar. Esto conlleva inconvenientes de socialización, se da una mutilación social y sensorial. Considero que este es otro aspecto sobre el que debemos indagar quienes nos interesamos en el tema.

\section{Internet y su influencia en la cultura.}

La integración de las tecnologías de la información en la sociedad ha venido provocando transformaciones profundas en su estructura económica y social. Uno de los campos donde su impacto es importante es el de la cultura. Es necesaria pues una lectura cultural de la comunicación en Internet, que nos permita acercarnos a las interacciones que se dan en la red.

$\mathrm{Al}$ abordar la manera en que debemos considerar las nuevas tecnologías de comunicación y su importancia para la vida social, Vidal nos dice que

Para comprender el significado de la información y la comunicación en la contemporaneidad debemos tratarlo como procesos complejos, interrelacionados con las restantes prácticas sociales e insertados en el entramado cultural. No se trata de analizar medios aislados que transmiten mensajes diversos, sino de evaluar en su integridad la articulación de estas prácticas con las restantes prácticas culturales en el sentido más amplio del término.

Estos procesos han alcanzado en la actualidad un marcado protagonismo en el funcionamiento social y se erigen como factor clave en el desarrollo y en la capacidad de éxito de las personas, las instituciones y los países. (2000: 52-53).

${ }^{2}$ Según Crovi, (2002) en México según cifras recientes alrededor de un millón de usuarios de Internet, que representa un número muy bajo en relación al total de la población, pero de gran influencia en la dinámica social.

${ }^{3}$ Los primeros resultados de una investigación que se desarrolla actualmente con jóvenes de la ciudad de Torreón nos hablan de que si bien en los primeros años de Internet le dedicaban buena parte de su tiempo libre, cada vez más ha pasado a ser un medio que no interfiere en sus actividades habituales. 
Por su parte, al hablar de la trascendencia de Internet en la vida actual, Castells afirma que "la comunicación a través del ordenador está revolucionando sin duda el proceso de comunicación, y por su mediación, la cultura en general" (2001b: 39 ). Un ejemplo de ello es que dentro de los segmentos de los usuarios habituales de Internet, al parecer el medio favorece la comunicación desinhibida y estimula la participación de trabajadores de posiciones inferiores dentro de las organizaciones. También es muy probable que las mujeres y otros sectores oprimidos de la sociedad se expresen con mayor franqueza a través de este medio.

Aunque el mayor uso de Internet se efectúa en situaciones de trabajo, más allá de tareas profesionales los usos de la comunicación mediada por computadora ya alcanzan toda la vida social. La actividad más habitual en este tipo de comunicación, fuera del trabajo, es el correo electrónico, cuya utilización sin embargo no sustituye a la comunicación interpersonal, sino a la telefónica. Otro uso creciente de la comunicación a través de la red es la política. El correo electrónico se está utilizando para la difusión masiva de propaganda de grupos de distintas tendencias.

Un fenómeno importante de la comunicación a través de Internet, en relación a la cultura, es la formación de comunidades virtuales, a las que se hace referencia en otra parte del trabajo. Por tratarse de un fenómeno aún reciente, todavía no se conoce cuanta sociabilidad existe en esas redes electrónicas y cuáles son sus efectos.

En lo que tiene que ver con el lenguaje de la comunicación mediada por computadora, algunos analistas consideran que, sobre todo el correo electrónico, representa el retorno de la mente tipográfica y el discurso racional y construido. Otros, por el contrario, consideran que la informalidad, espontaneidad y anonimato del medio estimulan una nueva forma de "oralidad". Esta última postura me parece más adecuada para describir lo que ocurre en la red. Sobre todo los jovenes han creado toda una gama de códigos que les facilita y les da mayor velocidad en la interacción.

\section{Nuevos patrones de interacción social.}

Desde el surgimiento de Internet, se ha dado un importante debate en torno a la aparición de nuevos patrones de interacción social y comunicativa. La formación de comunidades virtuales, por ejemplo, se ha interpretado como la culminación de un proceso histórico de disociación entre localidad y sociabilidad. De acuerdo con esta idea, 
nuevos modelos de relaciones sociales sustituyen las formas de interacción humana limitadas territorialmente. Por su parte, los críticos de Internet consideran que su expansión está conduciendo a un aislamiento social y una ruptura de la comunicación social y la vida familiar.

Para Castells (2001c) este debate adolece de tres limitaciones: su origen es anterior a la difusión generalizada de Internet, las opiniones expresadas se basaban en escasas experiencias de los primeros usuarios; se llevó a cabo en ausencia de información empírica sobre los usos reales de Internet; gira en torno a preguntas simplistas y engañosas, tales como la oposición entre una comunidad armoniosa en el pasado y una existencia alienada del internauta. Sobre esto último, el autor considera que el debate público sigue centrado en visiones simplistas que dificultan la comprensión de los nuevos modelos de interacción social.

De acuerdo con diversas investigaciones, los usos de Internet son fundamentalmente instrumentales y están relacionados con el trabajo, la familia y la vida cotidiana de los usuarios. El correo electrónico representa más del $85 \%$ del uso de Internet, y en su mayoría está relacionado con el trabajo, relaciones familiares y amigos en la vida real. Los chat rooms, los grupos de noticias y las conferencias resultaron significativas para los primeros usuarios, pero su importancia se redujo con la difusión de Internet. ${ }^{4}$ La construcción de identidad como base de la interacción on line representa una porción reducida de la sociabilidad basada en este medio, y es una actividad que tiende a concentrarse particularmente en adolescentes. No obstante,

la proliferación de estudios sobre esta cuestion ha hecho que se perciba a Internet como un terreno privilegiado para la práctica de las fantasías personales, cuando en realidad casi nunca lo es. Internet es una extensión de la vida tal como es, en todas sus dimensiones y modalidades. (139).

Frente a las opiniones encontradas en cuanto a lo que Internet significa para la sociabilidad, Castells señala que "parece ser que la interacción social en la red, en general, no tiene un efecto directo

Como se ha mencionado, la mayoría de las investigaciones sobre el tema han sido realizadas en los Estados Unidos. Sería importante conocer qué ocurre con los usos de Internet en nuestra realidad. 
sobre la configuración de la vida cotidiana, más allá de añadir la interacción on line a las relaciones sociales previamente existentes". (p. 140).

Internet es un medio que permite mantener los lazos sociales débiles. En ciertas condiciones, este medio puede crear nuevos tipos de lazos débiles, como ocurre en las comunidades de interés.

Algunas investigaciones (por ejemplo las de Barry Wellman, cit. por M. Castells), indican que el tipo de lazos que se crea a través de Internet son lazos débiles, pero sin embargo es un medio excelente para mantener y reforzar lazos que ya existían. La tendencia es hacia una disminución de la sociabilidad física tradicional, la relación en el barrio, la vida social en el trabajo. Se trata de un proceso de privatización de la sociabilidad, que se da no entre personas que trabajan o habitan en un mismo lugar, sino entre personas que construyen lazos electivos. También se ha encontrado que es un error la idea de que Internet es un lugar donde la gente solamente habla de frivolidades. Las comunidades virtuales tienen más éxito cuando están ligadas a tareas, a lograr intereses comunes, como las organizaciones de ayuda, redes religiosas, o de movilización social, como la de los globalifóbicos.

Internet puede contribuir también a mantener los lazos fuertes a distancia. "Pero el papel más importante de Internet en la reestructuración de las relaciones sociales es su contribución al nuevo modelo de sociabilidad, basado en el individualismo". (Castells 2001c: 151) Internet no crea un modelo de individualismo en red, pero proporciona el soporte material para la difusión del individualismo como forma dominante de sociabilidad.

Algunos investigadores han encontrado que la comunicación a través de la computadora y la comunicación telefónica son complementarias, particularmente en los contactos con amigos; los usuarios de Internet suelen tener menos contactos con sus familiares que los no usuarios. Estas diferencias se explican por la posición social: las personas con un status social superior con frecuencia tienen más amigos y que viven a mayor distancia, por lo que el correo electrónico se convierte en un instrumento que permite mantener la relación.

En cuanto a los efectos negativos de Internet para la sociabilidad, hay quienes consideran que este medio aísla, lleva a la depresión o incluso al suicidio, por el aislamiento de los individuos que pasan mucho tiempo navegando en la red. 


\section{Sociabilidad a través de los Chats.}

Una de las herramientas de Internet que ha venido a revolucionar las posibilidades de Interacción comunicativa es el chat. El chat presenta por lo menos dos características que lo diferencian de las interacciones "cara a cara": en primer término la carencia de información de un contexto social común a los sujetos involucrados en la interacción, en segundo que no es posible trasmitir o evidenciar las pautas no-verbales involucradas en la comunicación. (Roco, 2003).

Para Trejo, el correo electrónico imita al intercambio epistolar tradicional. "Pero hay nuevos usos de la red de redes que no parecen equiparables a otras formas de relación. El chat rompe con los parámetros habituales en el trato que los individuos pueden establecer en el espacio urbano" (2000: 5) La identidad queda supeditada a formas distintas de atracción y relación: empatía de ideas, simpatía mutua, entre otras.

Los sujetos que concurren a los chats son heterogéneos: sujetos de los más diversos lugares, culturas e ideologías, algo que no había sido logrado en otro sistema de comunicación. Quienes acceden a estos sitios en la red están dispersos por el mundo, y más allá de los límites que pueda significar el idioma, interactúan con otros sujetos con quienes no tendrían posibilidad de relacionarse o conocer a través de ningún otro medio de comunicación.

El chat es una instancia de reunión de individuos que interactúan, por tanto se comunican, forman y establecen relaciones interpersonales a escala mundial, lo que lleva a la interrogante de "cómo es posible la formación de lazos afectivos y relaciones íntimas, donde los contextos tradicionales de interacción han cambiado". (Roco, 2003).

Sánchez define el chat como:

la promoción no explícita de la fantasía; una cadena de sonidos que nunca llega al concierto total. Con su horario eterno que anula el sentido del tiempo y su carácter de metáfora, es la región que fascina pero atropella cualquier deseo de realidad. El chat es el lugar que deja sentir que ahí sólo tiene vida el tiempo libre. (1999: 21).

Si se compara al chat con el correo electrónico, el cual se considera más privado y confiable, las relaciones que se dan en éste último no tienen la emoción de las interacciones en vivo. 
Dentro de los motivos por los que la gente acude a los salones de chat, Cortázar (1998), señala tres: conocer a alguien para conversar, encontrar una pareja sentimental o establecer una relación sexual. No existe jerarquía entre ellos, ni tampoco son excluyentes.

Una de las características de Internet es que permite prácticas virtuales que se experimentan como reales. Este medio modifica hábitos que conducen a un cambio profundo en la construcción de la identidad o la psicología de los afectos. Para quienes frecuentan la red el ciberespacio es un lugar de gran significado, esencial para su existencia. En su trabajo, Sánchez busca:

mostrar cómo la red ha dado lugar a una globalización de los afectos, a una anulación del espacio y el tiempo que habla de un trastocamiento antropológico y del nacimiento de nuevas maneras de vivir la sensibilidad y las manifestaciones culturales. (2001: 14).

En este sentido, "El chat es ante todo un viaje, una forma de psicodelia y se caracteriza por su dinámica pop, de ahí que los jóvenes sean quienes de forma más afanosa y perseverante se conectan a tal medio". (p.25). Lo lúdico, la seducción por la amistad es lo que predomina, aunque existen chats sobre diversas cuestiones, como son culturales, filantrópicas, políticas o comerciales.

Las relaciones amorosas y los afectos se vuelven globales porque muchos chateadores entablan relaciones con personas de distintas partes del mundo. Esto es expresión del proceso de trastocamiento entre lo público y lo privado, propio de las sociedades contemporáneas. En los chats es posible participar en un área pública desde un ámbito privado. En la red, la globalización se convierte en realidad.

Como otras novedades de la realidad virtual, el chat tiene sus inconvenientes. Uno de ellos es que la gente que lo usa de manera asidua acaba por extrañar el contacto frente a frente.

Algo que debemos preguntarnos es por qué hay quienes prefieren interactuar con otros en el ciberespacio, frente a la posibilidad de hacerlo en la vida real. Para muchos es más fácil establecer relaciones a través de un medio donde la apariencia física no resulta tan importante. Los salones de chat estarían contribuyendo entonces a desarrollar el sentido de sociabilidad en personas que son tímidas en el mundo real.

En cuanto a su duración, en los chats las relaciones ya sea de amistad o afectivas se mantienen por tiempos variables, pueden ser efímeras, las que se dan sólo durante el tiempo de conexión o unos cuantos días; a mediano plazo, una relación que dura algunas semanas 
o unos cuantos meses y duraderas, las que se mantienen incluso por años.

Los salones de chat funcionan como lugar de encuentros, comunicación, creadores de identidad, que propician la formación de cibercomunidades, o comunidades virtuales. Es frecuente que quienes se conocen por primera vez en el chat hagan "cita" para reencontrarse de nuevo en el chat. De igual forma, las amistades que se establecen se prolongan más allá del chat, a través del envío de mensajes por e-mail, llamadas telefónicas o utilizando otros medios.

Por todo lo que se ha señalado, el chat es un aspecto importante a estudiar en la cibercultura, sobre todo porque es uno de los usos de Internet que más atraen a los jóvenes. Incluso hay quienes no hablan de "navegar", sino de "chatear".

\section{Comunidades virtuales.}

Una de las implicaciones socioculturales más importantes de la consolidación de Internet es la formación de las denominadas comunidades virtuales, nueva modalidad de organización que permite ponerse en contacto a un número ilimitado de usuarios interesados en distintas temáticas.

A partir de la creación de Internet, los individuos que se conectan a la red sienten que forman parte de un grupo o comunidad global, a diferencia de la etapa donde ya existían las telecomunicaciones, pero éstas eran utilizadas por individuos aislados. Las redes de comunicación se consideran hoy un fenómeno social, producto de un proceso de maduración tecnológica y cultural, que hace énfasis en el potencial que ofrecen para fomentar la comunicación, la cooperación y la comunidad, sin dejar de lado las posibilidades que brindan para la actividad comercial.

Sin embargo, como señala Pineda (2003), no debemos olvidar que si bien las redes facilitan las relaciones interpersonales, ellas por sí solas no pueden activar las relaciones comunicativas, son utilizadas por las personas para vincularse con otros y establecer nuevos modos de asociaciones humanas, a pesar de las distancias. La red nos permite interactuar con personas, organizaciones, comunidades y grupos lejanos, de distintas regiones y entornos sociales y culturales, y establecer relaciones de proximidad psicológica, cohesión en torno a intereses comunes:

De acuerdo con lo que propone Rheingold, Castells señala que por comunidades virtuales: 
suele entenderse una red electrónica autodefinida de comunicación interactiva, organizada en torno a un interés o propósito compartido, aunque a veces la comunicación se convierte en sí misma en la meta. Estas comunidades pueden estar relativamente formalizadas, como es el caso de la oferta de conferencias o los sistemas de tablones de anuncios, o formarse de modo espontáneo por redes sociales que siguen entrando en el sistema para enviar y recuperar mensajes con una pauta temporal elegida (ya sea en tiempo real o demorado). (2001b: $395)$.

Acerca del concepto de comunidades virtuales, Castells (2001c) señala que esta noción de los pioneros en el estudio de la sociabilidad a través de la red, tenía la virtud de hacer énfasis en el surgimiento de nuevos soportes tecnológicos para la sociabilidad, distintos pero no inferiores a las anteriores formas de interacción social. Sin embargo, con el término comunidad surgió una discusión ideológica entre los nostálgicos de la antigua comunidad, limitada espacialmente, y los partidarios de las comunidades electivas de Internet.

Para comprender las nuevas formas de interacción social en la era de Internet es necesario partir de una redefinición de la comunidad, haciendo menos énfasis en su componente cultural y subrayando la labor que cumple en cuanto a no limitar la existencia social de los individuos a una sola modalidad de acción material. Castells considera que se puede retomar la definición que propone Barry Wellman, "Las comunidades son redes de lazos interpersonales que proporcionan sociabilidad, apoyo, información, un sentimiento de pertenencia y una identidad social". (p.148).

Las comunidades virtuales desarrollan un espacio, un punto de congregación para individuos de intereses similares. Las comunidades virtuales inician una nueva dinámica en el surgimiento de ideas compartidas, los grupos de interés se conforman a partir de afinidades, de ahí que las nuevas tecnologías pueden ser pensadas como mediaciones en la construcción de un nuevo tipo de sociabilidad en la que el encuentro físico resulta irrelevante, aunque en muchos casos la relación que se inicia en el mundo virtual se extienda al mundo real.

A la pregunta de qué es lo que lleva a los individuos a entablar relaciones con congéneres digitales, Bermúdez y Martínez responden que "Quizá hallan descubierto algo maravilloso o una ilusión, lo posiblemente cierto es que estas tecnologías satisfacen de alguna 
manera la necesidad de contactos e intercambios de información que las barreras espaciales y sociales obstaculizan". $(1999$, p.4) Lo irónico, señalan en una visión crítica hacia estas nuevas formas de relación, es que las redes redefinen el espacio de lo social desde su propia lógica, dando como resultado múltiples interacciones de individuos sin referente espacial y temporal, acorpóreos, homogéneos. Y se preguntan: “¿Es ésta la clase de sujetos sociales producto de las comunicaciones mediadas por computadoras?"' (p.4) Considero que en esta postura hace falta tener en cuenta que en muchos casos relaciones que se inician de manera virtual y que logran cierta permanencia en el tiempo, pronto son complementadas con la comunicación telefónica, lo que da otra dimensión al vínculo, además de que se ha vuelto común el uso de cámaras que permiten tener acceso a la imagen de las personas con quienes interactuamos, aún en los sitios públicos que ofrecen servicio de Internet.

Existe una enorme diversidad de comunidades virtuales. Los usuarios por lo general tienden a transformar las nuevas tecnologías para satisfacer sus intereses y necesidades. A pesar de su diversidad, estas comunidades tienen como base dos características culturales compartidas de gran importancia: la primera de ellas es el valor de la comunicación horizontal y libre. Su actividad representa la práctica de la libertad de expresión a nivel global. Esta libertad es algo que los usuarios de la red defienden desde las primeras etapas de la comunicación on line. La segunda característica es lo que Castells denomina conectividad autodirigida, es decir la capacidad de cualquier persona para encontrar lo que le interesa en la red, y si no lo encuentra, para crear su propia información.

Según Benassini (1998), por la expansión de comunidades virtuales en torno a múltiples intereses y a su éxito como modalidad comunicativa, se les ha caracterizado como una nueva modalidad de comunicación interpersonal, en la medida en que supone intercambio de opiniones y puntos de vista con otros individuos. Considera que es importante tener en cuenta que se trata de una avanzada modalidad de comunicación interactiva, tomando en cuenta la distinción que establecen Betettini y Colombo entre interactividad -proceso mediante el que dos personas entran en contacto a través de la mediación tecnológica- e interacción, que supone el intercambio comunicativo cara a cara.

Acerca de la importancia del estudio de las comunidades virtuales, es posible señalar que la aparición de las comunidades mediadas por computadora es uno de los aspectos claves de investigación 
antropológica en la cibercultura. Este análisis es fundamental no sólo para comprender lo que son estas nuevas comunidades, sino también para imaginar el tipo de comunidades que los seres humanos somos capaces de crear con el apoyo de la tecnología.

Una de las cuestiones que se discuten en torno a esta nueva forma de relación social es si una tecnología como Internet destruye o construye comunidades. Por un lado hay quienes sostienen que Internet es un foro para la producción de comunidad, pero muchos están en desacuerdo, argumentando que por la misma naturaleza de la tecnología, la interacción a través de Internet se convierte en una actividad individual donde el "toque humano" es débil. Los tecnófobos argumentan que la tecnología es deshumanizante y por tanto su aplicación afectaría gravemente la cultura y la vida cotidiana. En mi opinión, la red representa un espacio que permite a quienes tenemos acceso a ella poder construir vínculos de acuerdo con nuestros intereses y afinidades, algo que en nuestro entorno cotidiano no siempre resulta fácil.

Un problema que se plantea para las relaciones humanas es el hecho de que se considera que existe una preferencia cada vez mayor por los contactos virtuales, más que por los reales. Esto debe llevarnos al cuestionamiento acerca del impacto que tendrán estas formas de interacción sobre las relaciones cara a cara; si las fronteras entre lo real y lo imaginario tendrán menos significación práctica; qué nuevas formas íntegras de relación y de hábitat pueden desarrollarse; qué sexualidad enfocaría una experiencia sexual en línea. (Campos, 2002). Este es sin duda un aspecto importante que debe considerarsẹ en la investigación sobre cibercultura. Lo que hemos observado en nuestro medio nos permite afirmar que no existe tal preferencia por los contactos virtuales, más bien son un complemento de los vínculos cara a cara, por ejemplo en el caso de los jóvenes estudiantes que se citan después de clase para "verse" en la red.

La idea de Internet como una enorme y diversa comunidad, ha llevado al concepto de ciudadanía de las redes, y preguntarnos si somos ciudadanos de las redes o la Internet es una red de ciudadanos. Estar en la red resulta un acto voluntario e individual. Se trataría pues de una ciudadanía que no tiene su origen en el nacimiento, sino de la decisión de estar en el ciberespacio. La ciudadanía en el ciberespacio carece del arraigo que tenemos en el mundo no virtual. En las redes experimentamos una ciudadanía nómada. (Trejo, 2000).

Para Quéau, (1995) las comunidades virtuales van a trastocar nuestra noción de vecindad; los “vecindarios" simbólicos y virtuales 
irán sustituyendo cada vez más a los vecindarios geográficos reales, que se basan en la proximidad fisica.

Las comunidades virtuales son verdaderas comunidades sociales, $\mathrm{y}$ los individuos que las componen establecen lazos afectivos, intercambian informaciones, colaboran entre sí. Esto es innegable. El problema surge de que estos lazos son virtuales, no están encarnados en cuerpos reales, sino sólo en clones simbólicos. (p. 77)

En relación a la trascendencia social de las comunidades virtuales, Kerkhove se pregunta: “¿Las ciudades virtuales son sólo un juguete o podemos esperar que surjan nuevas formas de socialización y comunidad de este tipo de experiencia?". (1999: 106) En los medios analógicos y digitales, es indispensable la interacción en tiempo real para que se produzca la sensación de presencia. Pero en los medios digitales es posible ampliar el espacio virtual y producir una sensación de que se pertenece a una comunidad virtual, es decir, se trata de una forma de "presencia" extendida.

\section{Relaciones afectivas en la red.}

En buena medida el interés que ha despertado Internet en los usuarios desde sus orígenes tiene que ver con la posibilidad de establecer relaciones afectivas a través de este medio. A diferencia de lo que muchos consideran, las comunicaciones en la red son bastante sinceras, por lo menos más de lo que supondríamos.

En el chat, con el texto como único medio de sensaciones y sentimientos, los enamoramientos se establecen con facilidad, las personas expresan con facilidad pensamientos que ocultarían en una relación cara a cara.

A través de investigaciones realizadas por Gwinell, (1999) se ha descubierto que mucha gente solamente logra experimentar un alto grado de confianza y de intimidad con personas que conocieron a través de Internet. La mayoría de ellos consideraban que la realidad física de los encuentros frente a frente restringía la intimidad.

Las relaciones afectivas en la red se inician como en el mundo real: a partir del interés mutuo y el conocimiento que se va dando entre quienes participan en ellas.

En las relaciones cara a cara, el nivel de cercanía requerido antes de que una pareja llegue al intercambio de información muy personal es variable. Hay quienes pueden hablar abiertamente sobre emociones 
profundas casi de inmediato, sin embargo la mayoría de la gente requiere de cierto tiempo de acercamiento antes de poder hablar de sus aspectos más íntimos. En la comunicación mediada por computadora aún no se cuenta con pautas de comportamiento que regulen el impacto de la comunicación con individuos que no conocemos físicamente.

La comunicación a través de la red facilita mucho la proyección de fantasías y sentimientos hacia personas desconocidas y a las que no hemos visto nunca. El anonimato crea una especie de efecto "máscara": la impresión de que se pueden decir cosas sin las restricciones de conducta y de lenguaje habituales. (p.p. 83-84).

La idea de que en las relaciones a través de Internet existe la sinceridad es muy común. Quienes están intercambiando correspondencia suelen dar por hecho que el otro es sincero, a pesar de que con frecuencia los internautas no dicen la verdad sobre ellos mismos. Sin embargo, cada vez es más frecuente que personas de todas las edades establezcan una relación afectiva con alguien que conocen en la red.

\section{A manera de conclusión.}

Después de revisar las ideas expuestas, acerca de Internet y la trascendencia de este medio para la vida social y para el campo de la comunicación, es posible afirmar que es en el terreno de la cultura donde es necesario profundizar en el conocimiento sobre la manera en que la red está cambiando las formas de relacionarnos. Es necesario dar respuesta a cuestionamientos como ¿cuáles son los usos que en nuestra realidad tiene Internet?, ¿Qué posibilidades ofrece Internet en cuanto a la interacción comunicativa?, ¿En qué medida la aparición de interacciones comunicativas a través de la red fortalece, debilita o modifica las relaciones comunicativas tradicionales?.

\section{Bibliografía.}

Benassini, C. (1998) De las Comunidades Sociales a las ¿Comunidades? Virtuales, (Notas para una discusión), Razón y Palabra Revista electrónica, 2 (3). Disponible en: http:// www.razonypalabra.org.mx.

Campos, J. L. (2002). Comunicación, comunidades y prácticas culturales en la cibercultura. Razón y Palabra Revista electróni- 
ca, 27 (7)

Castells, M. (2001, mayo) Internet y la sociedad red. Etcétera, 4962.

(2001). La era de la información. Economía, Sociedad y Cultura. (I) La sociedad red. ( $3^{a}$. Ed.). México: Siglo XXI. (2001). La galaxia Internet. Reflexiones sobre

Internet, empresa y sociedad. España: Areté.

Cortázar, F. (1998) Ciberrelaciones: amistad, amor y sexo en los salones del chat. Comunicación y Sociedad, (34), 129-154.

Crovi, D. (2002) Encuentros y desencuentros en el ciberespacio. Versión (12), 257-270.

E. Bermúdez y G. Martínez. Identidades colectivas en el ciberespacio. Interrogantes más que respuestas. Disponible en: http://www.innovarium/innovarium.com/rev105/ identidades\%20digitales.htm consultado abril 2003.

La sociedad del conocimiento en México. (2001, febrero) Este País (119), 59-60.

Fuentes Navarro, R. (2001) Exploraciones teórico-metodológicas para la investigación sociocultural de los usos de la Internet", en M. Vasallo y R. Fuentes Navarro (comps.). Comunicación.

Campo y objeto de estudio. Perspectivas reflexivas latinoamericanas. México: ITESO.

Galindo, J. (1998). Redes, Comunidad Virtual y Cibercultura, Razón y Palabra Revista electrónica, 10, (3). Disponible en: http://www.razonypalabra.org.mx

González Manet, E. (1998). Internet: espejismos y promesas de la cultura electrónica. Bimestre Cubana, (9), 98-117. (2000, enero-junio). Cultura, globalización y nuevas tecnologías de comunicación. Temas. (20/21), 4-11. Gubern, R. (2000). El eros electrónico, México: Taurus. Gwinell, E. (1999). El amor en Internet. Intimar con desconocidos a través del ciberespacio. España: Paidós.

Joyanes, L. ( 1997). Cibersociedad. Los retos sociales ante un nuevo mundo digital. España: Mc Graw Hill.

Kerkhove, D. (1999). Inteligencias en conexión. España: Gedisa. Negroponte, N. (1995). Ser Digital, México: Océano.

Pineda de Alcázar, M. (2003, enero-junio). El papel de Internet como un nuevo medio de comunicación social en la era digital. Hiper-textos (6). Disponible en http://hiper-textos.mty.itesm.mx Quéau, P. (1995). Lo virtual. Virtudes y vértigos. España: Paidós. 
Ramonet, I. (2001, septiembre). Por sí misma, Internet no cambia el mundo. Etcétera.

Disponible en: http://www.etcetera.com.mx

Roco, G. Internet y Sociedad: un fenómeno emergente. Disponible en:

http://ubiobio.cl/eps/ponencia/doc/p.1.1 htm consultado en julio 2003.

Sánchez, A. (1999). El chat. Etcétera, primera época, mayo.

Disponible en http://www.etcetera.com.mx (2001). La era de los afectos en Internet. México:

\section{Océano.}

Trejo D., Raúl. (2002). Internet es el futuro. Telos, (51), segunda época, abril-junio. Disponible en: http://campus.red/telos/ (2002). La gran conversación. Etcétera. Noviembre. Disponible en http://www.etcetera.com.mx

Vidal, J.R. (2000, enero-junio). La clave está en el conocimiento. Temas. (20/21), 51-55.

Wolton, D. (1999). Internet ¿y después?, España: Gedisa. 